\title{
Macrophage adaptation in airway inflammatory resolution
}

\author{
Manminder Kaur, Thomas Bell, Samira Salek-Ardakani and Tracy Hussell
}

Affiliation: Manchester Collaborative Centre for Inflammation Research, Manchester University,
Core Technology Facility, Manchester, UK.

Correspondence: Tracy Hussell, Manchester Collaborative Centre for Inflammation Research, Manchester University, Core Technology Facility, Grafton Street, Manchester, M13 9NT, UK.

E-mail: tracy.hussellamanchester.ac.uk

ABSTRACT Bacterial and viral infections (exacerbations) are particularly problematic in those with underlying respiratory disease, including post-viral infection, asthma, chronic obstructive pulmonary disease and pulmonary fibrosis. Patients experiencing exacerbations tend to be at the more severe end of the disease spectrum and are often difficult to treat. Most of the unmet medical need remains in this patient group. Airway macrophages are one of the first cell populations to encounter airborne pathogens and, in health, exist in a state of reduced responsiveness due to interactions with the respiratory epithelium and specific factors found in the airway lumen. Granulocyte-macrophage colony-stimulating factor, interleukin-10, transforming growth factor- $\beta$, surfactant proteins and signalling via the CD200 receptor, for example, all raise the threshold above which airway macrophages can be activated. We highlight that following severe respiratory inflammation, the airspace microenvironment does not automatically re-set to baseline and may leave airway macrophages more restrained than they were at the outset. This excessive restraint is mediated in part by the clearance of apoptotic cells and components of extracellular matrix. This implies that one strategy to combat respiratory exacerbations would be to retune airway macrophage responsiveness to allow earlier bacterial recognition.

@ERSpublications

Re-educating airway macrophages that do not reset to baseline following severe inflammation http://ow.ly/NeMmM

\section{Introduction}

Airway macrophages are $95 \%$ pure in the healthy murine and human lung. These sentinel cells must select contradictory responses which are dependent on the requirement of the tissue at that time; such as inflammation to pathogens, or anti-inflammatory responses when clearing self-apoptotic cells and components of extracellular matrix turnover and degradation. Their function is exquisitely tuned by the airspace microenvironment which is rich in soluble (granulocyte-macrophage colony-stimulating factor (GM-CSF), interleukin (IL)-10, transforming growth factor (TGF)- $\beta$ ) and surfactant proteins and contact-dependent (CD200) immune-regulatory signals. Macrophages colonise the airways shortly after birth, originating from fetal monocytes [1], and are particularly long-lived compared to those from other sites $[2,3]$. Due to current technical limitations in the ability to trace lineage, it is difficult to ascertain the percentage of turnover of tissue-resident macrophages. In addition, alveolar macrophages have a remarkable capacity for self-renewal, and it is suggested that they are replenished from circulating monocytes following their ablation by severe irradiation [4-7] or infection [8]. Alveolar macrophages are also unusual in that they express a unique repertoire of receptors [9]. These include CD11c, which is an integrin that is otherwise only expressed on mucosal macrophages such as those found in the gut [10],

Received: April 022015 | Accepted after revision: May 092015

Conflict of interest: Disclosures can be found alongside the online version of this article at err.ersjournals.com.

Provenance: Submitted article, peer reviewed.

Copyright OERS 2015. ERR articles are open access and distributed under the terms of the Creative Commons Attribution Non-Commercial Licence 4.0. 
SiglecF, which has only previously been described on eosinophils [11] and Axl, which is not found on other macrophage populations [12].

The activation of alveolar macrophages must be tightly regulated to prevent unwanted immune responses to innocuous inhaled antigens, and this is mediated by both soluble factors in the lumen of the airways and through cell-cell interactions [9]. The dominant cell-cell interactions in the healthy airways are between alveolar macrophages and respiratory epithelial cells, including the bronchial epithelium as well as type I and type II alveolar epithelial cells, which can exert a range of immunomodulatory stimuli over macrophages. The human bronchial epithelial cell line BEAS-2B restricts alveolar macrophage responses in vitro through both cell contact- and soluble factor-mediated interactions [13] and exemplifies the complex crosstalk between the epithelium and luminal macrophages.

\section{Negative regulators of alveolar macrophages}

Alveolar macrophages are highly regulated to prevent unwanted inflammatory responses to innocuous inhaled antigens. This is achieved through soluble factors such as IL-10 and surfactant proteins A and D, as well as physical interactions between alveolar macrophages and the respiratory epithelium via CD200R and $\alpha v \beta 6$-tethered TGF- $\beta$. Interactions between hyaluronan and CD44 may also inhibit Toll-like receptor (TLR) signalling through the induction of the TLR-negative regulators A20 and IL-1 receptor-associated kinase (IRAK)-M.

Little is known about airway macrophage heterogeneity or regulation following inflammatory resolution. Macrophages are significantly depleted during influenza infection. Replenishment occurs from the interstitial lung macrophage pool rather than from blood monocytes [14]. Though this return to immune homeostasis leaves an airway macrophage population essentially phenotypically identical to those at the beginning $\left(\mathrm{CD} 11 \mathrm{c}^{+}, \mathrm{CD} 11 \mathrm{~b}\right.$ intermediate, $\mathrm{F} 4 / 80^{+}$, Siglec $\left.\mathrm{F}^{+}\right)$, their responsiveness is significantly dampened [15]. We referred to this inhibition as "innate imprinting" in 2004 [16], but more recently the concept of "trained immunity" has been proposed, whereby monocytes acquire a tolerant phenotype after stimulation that is associated with a switch from oxidative metabolism to glycolysis $[17,18]$. Interestingly, this refractory airway macrophage response exists across a wide spectrum of pathologies (post-viral, allergic, fibrotic, etc.) in mice and humans, which implies that it is not driven by the "antigen" or immune response to it, but by specific features of the repairing lung.

\section{Infection risk}

The spectrum of pathologies in which we identified a refractory macrophage phenotype is coincidentally associated with recurring bacterial complications (exacerbations) leading to increased sepsis, hospitalisation, morbidity and death, and accounts for some of the most prevalent diseases in the world today. These patients account for a large fraction of overall cost, and most of the unmet need remains at the severe end of the disease spectrum. Despite the diversity in pathology, the bacterial species responsible for exacerbation of disease are remarkably reproducible, suggesting a common underlying deficient process(es). This is irrespective of the initial causative agent or the immune response induced.

Respiratory viral infection often causes bacterial complications that increase disease morbidity [8, 19, 20]. Bacterial species such as Streptococcus pneumoniae, Staphylococcus aureus and Haemophilus influenzae normally exist commensally within the lung, but can act as opportunistic pathogens during viral infection [21]. These secondary bacterial infections are sometimes termed superinfections, and are a leading cause of increased mortality following viral infection. During the 1918 "Spanish flu" and 1957 "Asian flu" pandemics, up to $20 \%$ of patients infected with influenza also harboured a secondary bacterial infection [22]. Post-mortem examinations revealed that during the 1918 pandemic $>93 \%$ of deaths were associated with bacterial complications [23]. Similarly, a retrospective study of medical records from various United States army bases described an onset of bacterial infection 7-10 days after initial influenza infection in $\sim 20 \%$ of patients [24]. In addition, during the $2009 \mathrm{H} 1 \mathrm{N1}$ pandemic, secondary bacterial infections accounted for $20-41 \%$ of deaths $[25,26]$. While not as prevalent during seasonal influenza, bacterial co-infections still account for $40 \%$ of all respiratory viral infections resulting in hospitalisation [27].

Various animal infection models have confirmed the link between respiratory virus infection and susceptibility to secondary bacterial superinfection, but the complex underlying mechanisms remain poorly understood $[28,29]$. It is likely that physical damage to the lung itself, long-lasting changes to innate immunity and synergistic interactions between virally infected host cells and bacteria all play a role in susceptibility to bacterial infection.

Mechanisms of susceptibility to infections

Whatever the mechanism following severe inflammation, negative interactions between alveolar macrophages and the airway epithelium increase. CD200R on alveolar macrophages and IL-10 are 
increased, both of which provide strong inhibitory signals $[30,31]$ and lead to heightened susceptibility to bacterial infections [32].

Overproduction of interferon- $\gamma$ during viral infection impairs the phagocytosis of bacteria by alveolar macrophages, and reduces their expression of the bacterial scavenger receptor MARCO (macrophage receptor with collagenous structure) [33], and similarly the presence of type I interferons generated during influenza infection can limit antibacterial immunity [34]. Alveolar macrophage TLR responses are restricted for several months following the resolution of influenza, highlighted by reduced nuclear translocation of nuclear-factor (NF)- $\mathrm{KB}$ in response to the TLR5 ligand flagellin [15]. Depleting and regenerating the resident macrophage pool after viral infection restores antibacterial immunity [15], highlighting the importance of alveolar macrophages in the susceptibility to bacterial complications. Asthmatic patients can also suffer from bacterial complications, and increased susceptibility to bacterial infection is observed in mouse models of allergic airway disease. As with viral infections, this is associated with increased expression of the negative regulators such as CD200R on alveolar macrophages [35] (fig. 1).

Another contributing generic process relevant to all pulmonary inflammatory conditions is the need to remove apoptotic cells before they undergo secondary necrosis. The Tyro3, AXL and MerTK (TAM) receptor tyrosine kinase family [36] recognise phosphatidylserine on apoptotic cells via the bridging molecules protein $S$ and growth arrest specific (GAS)-6 proteins. TAM receptors are expressed on phagocytic cells [36,37] and inhibit inflammation during apoptotic cell efferocytosis via a negative feedback loop involving activation of suppressor of cytokine signalling-1 and -3 that in turn inhibit cytokine and Toll-like receptor signalling pathways [38-40]. Although inhibition of innate inflammation is essential to prevent autoimmunity during apoptotic cell clearance, prolonged engagement of TAM receptors may cause a state of unresponsiveness in antigen presenting cells required to clear pathogenic micro-organisms. To support this hypothesis, elevated GAS-6 plasma levels are observed in patients with severe sepsis [41, 42], and MerTK is elevated on monocytes from patients with septic shock, and is linked to an adverse outcome [43]. The consequences of these observations remain as yet unexplored. We have

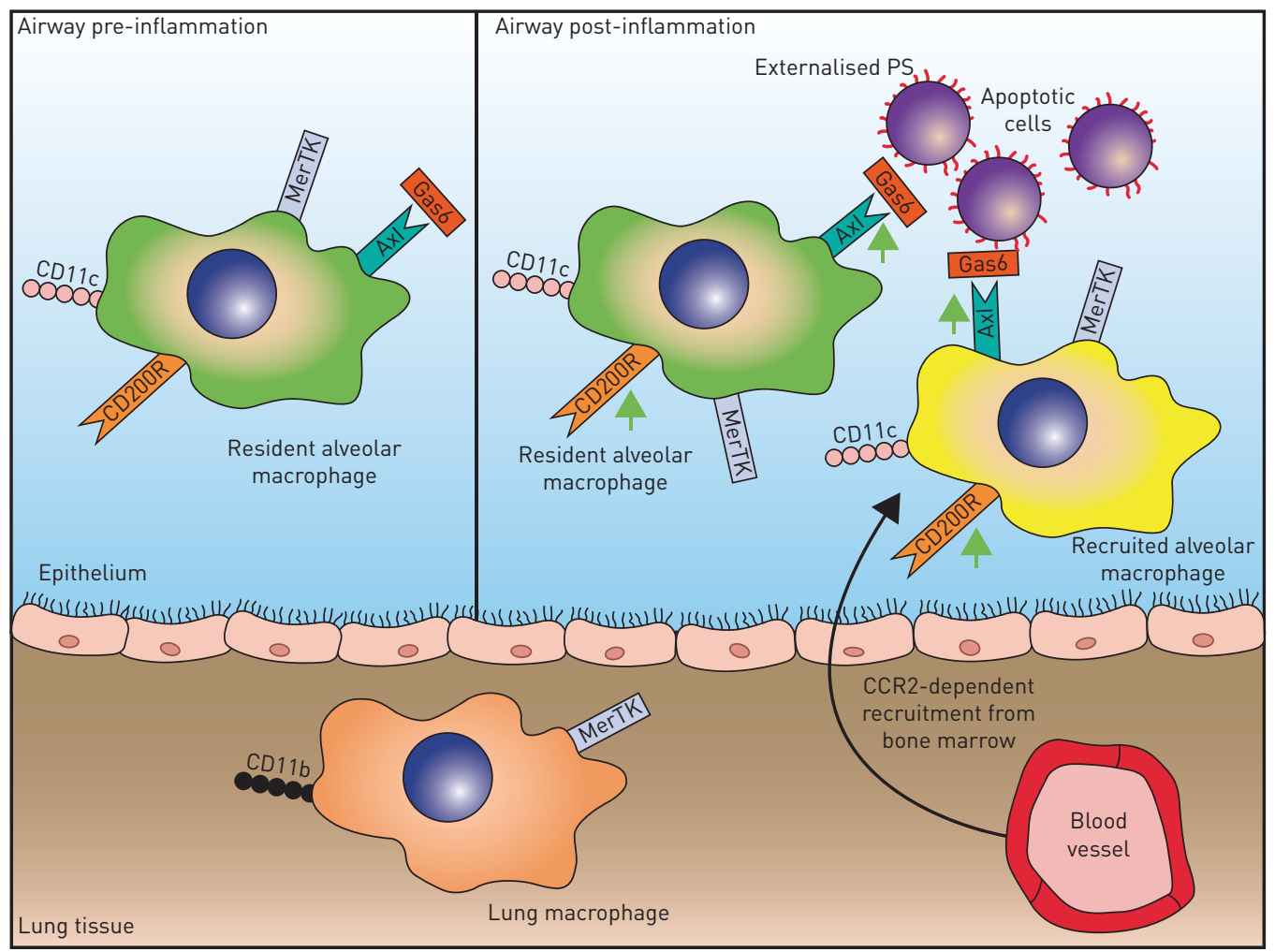

FIGURE 1 Resident alveolar macrophages in health (green) are influenced by the airway microenvironment to express a unique phenotype high in regulatory receptors that prevent responses to innocuous antigens. During inflammation airway macrophages are depleted and may be replaced in resolution by recruited monocytes that differentiate to macrophages and adapt to the airspace microenvironment (yellow). Generic processes include removal of extracellular matrix and efferocytosis of apoptotic cells. GAS: growth arrest specific protein; PS: phosphatidylserine; CCR: chemokine receptor. 


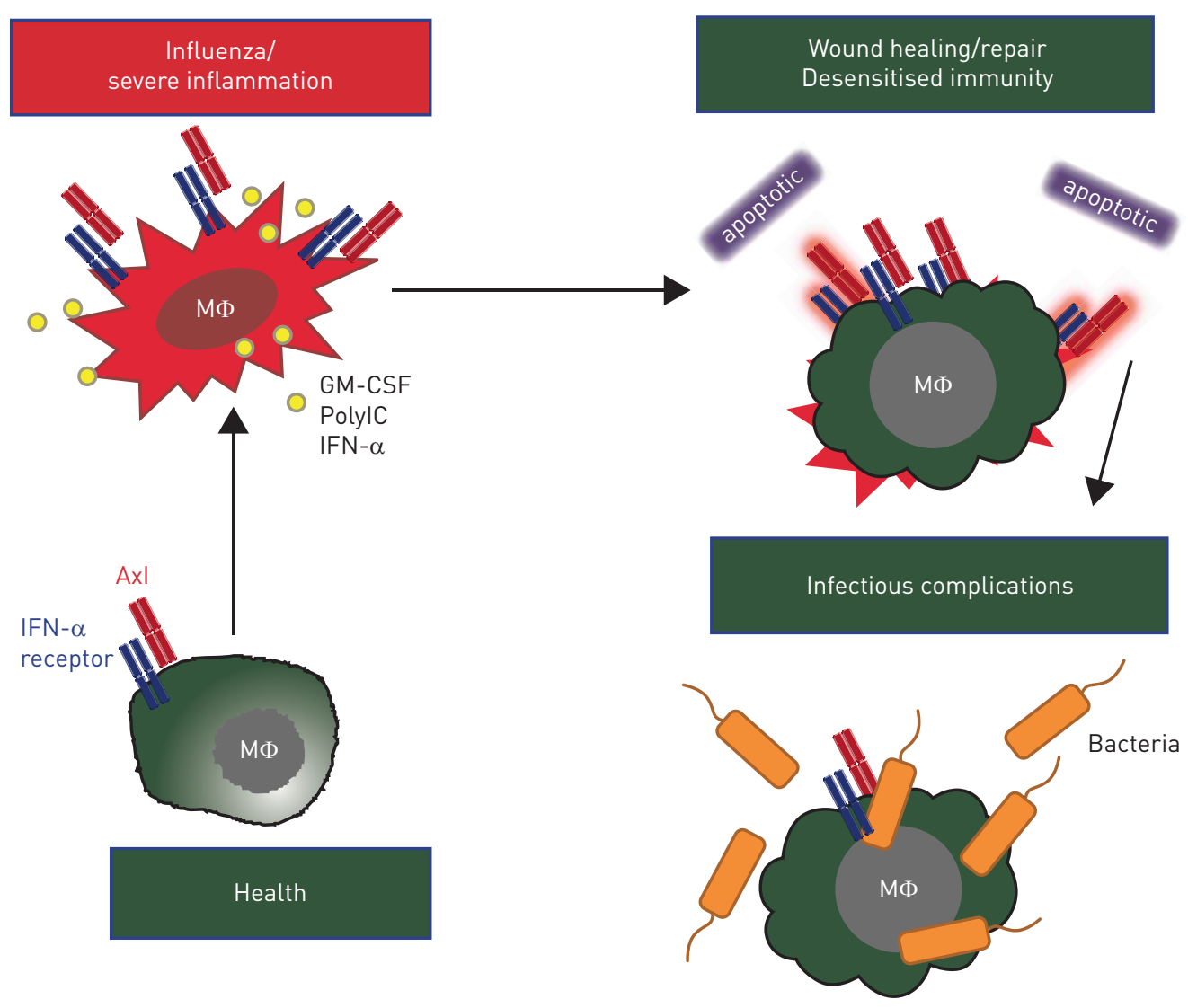

FIGURE 2 Axl is constitutively expressed on murine airway macrophages (MФ). Granulocyte-macrophage colony-stimulating factor (GM-CSF), polyinosinic-polycytidylic acid (polyIC) or interferon (IFN)- $\alpha$ upregulate Axl expression to mediate efferocytosis of apoptotic cells. Axl signalling induces suppressor of cytokine signalling-1 and -3 which impair tumour necrosis factor receptor associated factor (TRAF)3, TRAF6 and Mal, required for Toll-like receptor-4 signalling. This prevents autoimmunity to self-proteins. However, ongoing removal of apoptotic cells causes macrophage insensitivity to subsequent bacterial infection.

published several novel concepts that relate to TAM receptor function in the lung: 1) in health AXL is exclusively expressed on airway macrophages due to the presence of high levels of GM-CSF; 2) AXL-lacking human monocyte-derived macrophages (MDMs) can be induced to express AXL in the presence of GM-CSF; and 3) unlike AXL, the bridging molecule GAS-6 is exclusively expressed in macrophage-CSF-driven MDMs. It thus appears that macrophages either express AXL (and efferocytose apoptotic cells) or produce GAS-6 (and assist other cells in efferocytosis). This suggests a previously undiscovered functional dichotomy in macrophage subsets and that only those expressing AXL may be blunted in their response to bacteria (fig. 2).

Another process common to all lung inflammatory conditions is the alteration of the matrisome through damage and repair of extracellular matrix. Similar to apoptotic cell efferocytosis, clearance of self-matrix requires processes to prevent inadvertent autoimmune reactions. Damaged extracellular matrix provides cues to surrounding cells to drive a protective response. In a dysregulated state, the excess production of a pathological matrix is likely to contribute to chronic inflammatory conditions, aberrant airway macrophage training and subsequent bacterial exacerbation.

\section{Concluding remarks}

There is a real possibility that generic treatments may alleviate exacerbations common in inflammatory lung conditions. An over-regulated airway macrophage will not be able to alert the presence of a pathogenic micro-organism quickly. Thus, replacement of the initial mediators produced may be one strategy. This is supported by our observation that instillation of the neutrophil chemoattractant KC (keratinocyte chemoattractant) restores neutrophil recruitment and reduces bacterial load in allergic mice by several logs [35]. Alternatively, the fact that airway macrophages are so long lived presents a problem in that if they are over-regulated they will not be able to respond to a pathogenic micro-organism quickly. This suggests that macrophage replenishment may also be beneficial, i.e. repopulating the pool with less 
regulated macrophages so that they are more responsive and able to sense more readily a pathogenic micro-organism. However, this assumes that the defect or alteration is within the macrophage, but we believe the change is in the repaired epithelium/matrix and so any incoming macrophage would be similarly affected. Finally, extracellular matrix components, such as hyaluronan, dominates the airspace matrisome in a number of situations. This hyaluronan can be complexed to other self-proteins and, in some cases, assume the functions of that adopted protein. For example, the hyaluronan complexes with heavy chains derived from inter- $\alpha$ inhibitor catalysed by TNF-stimulated gene 6 [44]. The complex of inter- $\alpha$ inhibitor with heavy chains functions to limit complement-mediated phagocytosis [45] and so it is tempting to speculate that this function is transferred to hyaluronan during inflammation. Discovering the molecular mechanisms of macrophage adaptation in the airspaces has the potential to open entirely new avenues amenable to therapeutic intervention that target the host.

\section{References}

1 Guilliams M, De Kleer I, Henri S, et al. Alveolar macrophages develop from fetal monocytes that differentiate into long-lived cells in the first week of life via GM-CSF. J Exp Med 2013; 210: 1977-1992.

2 Maus UA, Janzen S, Wall G, et al. Resident alveolar macrophages are replaced by recruited monocytes in response to endotoxin-induced lung inflammation. Am J Respir Cell Mol Biol 2006; 35: 227-235.

3 Janssen WJ, Barthel L, Muldrow A, et al. Fas determines differential fates of resident and recruited macrophages during resolution of acute lung injury. Am J Respir Crit Care Med 2011; 184: 547-560.

4 Sawyer RT, Strausbauch PH, Volkman A. Resident macrophage proliferation in mice depleted of blood monocytes by strontium-89. Lab Invest 1982; 46: 165-170.

5 Schmidt A, Sucke J, Fuchs-Moll G, et al. Macrophages in experimental rat lung isografts and allografts: infiltration and proliferation in situ. J Leukoc Biol 2007; 81: 186-194.

6 Tarling JD, Lin HS, Hsu S. Self-renewal of pulmonary alveolar macrophages: evidence from radiation chimera studies. J Leukoc Biol 1987; 42: 443-446.

7 Yona S, Kim KW, Wolf Y, et al. Fate mapping reveals origins and dynamics of monocytes and tissue macrophages under homeostasis. Immunity 2013; 38: 79-91.

8 McCullers JA, McAuley JL, Browall S, et al. Influenza enhances susceptibility to natural acquisition of and disease due to Streptococcus pneumoniae in ferrets. J Infect Dis 2010; 202: 1287-1295.

9 Hussell T, Bell TJ. Alveolar macrophages: plasticity in a tissue-specific context. Nat Rev Immunol 2014; 14: 81-93.

10 Zaynagetdinov R, Sherrill TP, Kendall PL, et al. Identification of myeloid cell subsets in murine lungs using flow cytometry. Am J Respir Cell Mol Biol 2013; 49: 180-189.

11 Misharin AV, Morales-Nebreda L, Mutlu GM, et al. Flow cytometric analysis of macrophages and dendritic cell subsets in the mouse lung. Am J Respir Cell Mol Biol 2013; 49: 503-510.

12 Fujimori T, Grabiec AM, Kaur M, et al. The Axl receptor tyrosine kinase is a discriminator of macrophage function in the inflamed lung. Mucosal Immunol 2015 [In press DOI: 10.1038/mi.2014.129].

13 Mayer AK, Bartz H, Fey F, et al. Airway epithelial cells modify immune responses by inducing an anti-inflammatory microenvironment. Eur J Immunol 2008; 38: 1689-1699.

14 Ghoneim HE, Thomas PG, McCullers JA. Depletion of alveolar macrophages during influenza infection facilitates bacterial superinfections. J Immunol 2013; 191: 1250-1259.

15 Didierlaurent A, Goulding J, Patel S, et al. Sustained desensitization to bacterial Toll-like receptor ligands after resolution of respiratory influenza infection. J Exp Med 2008; 205: 323-329.

16 Williams AE, Edwards L, Humphreys IR, et al. Innate imprinting by the modified heat-labile toxin of Escherichia coli (LTK63) provides generic protection against lung infectious disease. J Immunol 2004; 173: 7435-7443.

17 Saeed S, Quintin J, Kerstens HH, et al. Epigenetic programming of monocyte-to-macrophage differentiation and trained innate immunity. Science 2014; 345: 1251086.

18 Cheng SC, Quintin J, Cramer RA, et al. mTOR- and HIF- $1 \alpha$-mediated aerobic glycolysis as metabolic basis for trained immunity. Science 2014; 345: 1250684.

19 McCullers JA. Insights into the interaction between influenza virus and pneumococcus. Clin Microbiol Rev 2006; 19: $571-582$.

20 Lehtinen P, Jartti T, Virkki R, et al. Bacterial coinfections in children with viral wheezing. Eur J Clin Microbiol Infect Dis 2006; 25: 463-469.

21 Hament JM, Kimpen JL, Fleer A, et al. Respiratory viral infection predisposing for bacterial disease: a concise review. FEMS Immunol Med Microbiol 1999; 26: 189-195.

22 McCullers JA, Rehg JE. Lethal synergism between influenza virus and Streptococcus pneumoniae: characterization of a mouse model and the role of platelet-activating factor receptor. J Infect Dis 2002; 186: 341-350.

23 Morens DM, Taubenberger JK, Fauci AS. Predominant role of bacterial pneumonia as a cause of death in pandemic influenza: implications for pandemic influenza preparedness. J Infect Dis 2008; 198: 962-970.

24 Brundage JF. Interactions between influenza and bacterial respiratory pathogens: implications for pandemic preparedness. Lancet Infect Dis 2006; 6: 303-312.

25 Bautista E, Chotpitayasunondh T, Gao Z, et al. Clinical aspects of pandemic 2009 influenza A (H1N1) virus infection. N Engl J Med 2010; 362: 1708-1719.

26 Lucas S. Predictive clinicopathological features derived from systematic autopsy examination of patients who died with A/H1N1 influenza infection in the UK 2009-10 pandemic. Health Technol Assess 2010; 14: 83-114.

27 Falsey AR, Becker KL, Swinburne AJ, et al. Bacterial complications of respiratory tract viral illness: a comprehensive evaluation. J Infect Dis 2013; 208: 432-441.

28 Habibzay M, Weiss G, Hussell T. Bacterial superinfection following lung inflammatory disorders. Future Microbiol 2013; 8: 247-256.

29 McCullers JA. The co-pathogenesis of influenza viruses with bacteria in the lung. Nat Rev Microbiol 2014; 12: 252-262. 
30 van der Sluijs KF, van Elden LJ, Nijhuis M, et al. IL-10 is an important mediator of the enhanced susceptibility to pneumococcal pneumonia after influenza infection. J Immunol 2004; 172: 7603-7609.

31 Snelgrove RJ, Goulding J, Didierlaurent AM, et al. A critical function for CD200 in lung immune homeostasis and the severity of influenza infection. Nat Immunol 2008; 9: 1074-1083.

32 Goulding J, Godlee A, Vekaria S, et al. Lowering the threshold of lung innate immune cell activation alters susceptibility to secondary bacterial superinfection. J Infect Dis 2011; 204: 1086-1094.

33 Sun K, Metzger DW. Inhibition of pulmonary antibacterial defense by interferon- $\gamma$ during recovery from influenza infection. Nat Med 2008; 14: 558-564.

34 Shahangian A, Chow EK, Tian X, et al. Type I IFNs mediate development of postinfluenza bacterial pneumonia in mice. J Clin Invest 2009; 119: 1910-1920.

35 Habibzay M, Saldana JI, Goulding J, et al. Altered regulation of Toll-like receptor responses impairs antibacterial immunity in the allergic lung. Mucosal Immunol 2012; 5: 524-534.

36 Lemke G, Rothlin CV. Immunobiology of the TAM receptors. Nat Rev Immunol 2008; 8: 327-336.

37 Linger RM, Keating AK, Earp HS, et al. TAM receptor tyrosine kinases: biologic functions, signaling, and potential therapeutic targeting in human cancer. Adv Cancer Res 2008; 100: 35-83.

38 Alciato F, Sainaghi PP, Sola D, et al. TNF- $\alpha$, IL-6, and IL-1 expression is inhibited by GAS6 in monocytes/ macrophages. J Leukoc Biol 2010; 87: 869-875.

39 Rothlin CV, Lemke G. TAM receptor signaling and autoimmune disease. Curr Opin Immunol 2010; 22: 740-746.

40 Sharif MN, Sosic D, Rothlin CV, et al. Twist mediates suppression of inflammation by type I IFNs and Axl. J Exp Med 2006; 203: 1891-1901.

41 Ekman C, Linder A, Akesson P, et al. Plasma concentrations of Gas6 (growth arrest specific protein 6) and its soluble tyrosine kinase receptor sAxl in sepsis and systemic inflammatory response syndromes. Crit Care 2010; 14: R158.

42 Borgel D, Clauser S, Bornstain C, et al. Elevated growth-arrest-specific protein 6 plasma levels in patients with severe sepsis. Crit Care Med 2006; 34: 219-222.

43 Guignant C, Venet F, Planel S, et al. Increased MerTK expression in circulating innate immune cells of patients with septic shock. Intensive Care Med 2013; 39: 1556-1564.

44 Milner CM, Day AJ. TSG-6: a multifunctional protein associated with inflammation. J Cell Sci 2003; 116: 1863-1873.

45 Okroj M, Holmquist E, Sjölander J, et al. Heavy chains of inter alpha inhibitor (I $\alpha$ I) inhibit the human complement system at early stages of the cascade. J Biol Chem 2012; 287: 20100-20110. 